Ferner fand sich fast regelmäßig eine Zunahme der Spannung des Pulses, und zwar unter Abnahme seines Volumens, wenn dieses vorher gro B war, wie namentlich bei einem Falle von Aorteninsuffizienz. Es wnirde also der Tonus der Arterien erhöht! Wie sich dieses bei arteriospastischen Zuständen verhält, hatte ich keine Gelegenheit zu beobachten.

Was die interessante Frage nach der Beeinflussung der Herzgröße durch das einzelne Bad anlangt, so konnte ich in einigen Fällen von perkutorisch sicher nachweisbarer Dilatation ein geringes Zurückgehen derselbell nach dem Bade konstatieren; besonders objektiv lehrreich ist in dieser Hinsicht ein Fall von Aorteninsuffizienz gewesen mit starkem, verbreitertem und bedeutend nacl links verlagertem Spitzenstoß; in diesem Falle konnte man nämlich dic linke Grenze des Herzens in der Gegend des Spitzenstoßes genau abtasten, sodaß eine Täuschung ausgeschlossen ist; hier zeigte sich nun nach dem Bade der Spitzenstol weniger hebend und breit und etwa $1 \mathrm{~cm}$ nach innen gerückt; es kann dies wohl nur auf besserer Kontraktion der Ventrikel und Abnahme der bestehenden Dilatation beruhen.

Die bei den $\mathrm{CO}_{2}$-haltigen Thermalsolbädern so dentliche Reaktion, d. h. die Erweiterung der Kapillaren der Hant, fehlt bei den Wechselstrombädern vollständig; der Welt der Reaktion geht aus der physiologischen Tatsache hervor, daß3 die Strömungsschnelligkeit in einer Gefäßstrecke dem Unterschied des Druckes im Querschnitt des Anfangs und des Endes dieser Bahnstrecke entspricht. Dagegen scheint der Einflı $\beta$ des Wechselstroms auf den Tonns der Gefälie bei angioparalytischen Zuständen ein größ3erer zu sein. Es erscheint daher rationell, bei rewissen Fällen eine Kombination beider Methoden, nämlich der Wechselstrombäder und der Nauleimer Bäder anzuwenden, worauf ich schon ror einem Jahre hinwies.

Wie ibberall in der Elektrophysiologie kommen natürlich auch hier bestimmte Wirkungen nur entsprechenden Stromstärken zu; dieses gilt besonders vom Vasomotorenzentrum in der Medulla. um dessen reflektorische Erregung es sich hiel wohl hauptsächlich handelt; aus der Physiologie wissen wir, dab schwache elektrische Reize pressorisch, starke depressorisch larauf wirken.

Endlich möclite ich noch erwähnen, dal3 ınir der dreiphasige sinusoüdale Wechselstrom eine etwas stärkere Wirkung als der einphasige zu haben scheint.

Znm Schluß gestatte ich mir noch, dem Oberalzt am hiesigen Konitzky-Stift, Herrn Oberstabsarzt Dr. Steinber $\mathrm{g}$, meinen verbindlichsten Dank für gütiges Entgegenkommen bei der Ausfülırung meiner Untersuchungen allszusprechen.

\section{Einige Beobachtungen über die Wirkung der sinusoïdalen Wechselstrombäder auf die Zirkulationsorgane.} Voriäufige Mitteilung von Dr. Paul C. Franze in Bad Nauheim.

Im Nachfolgenden gestatte ich mir, kurz über einige Untersuchungen, die ich in der letzten Zeit anstellte, $1 \mathrm{~m}$ die Wirkung sinusoidaler Wechselstrombäder auf Herz und Gefäße zu ermitteln, zu berichten.

Die Anordnung der Versinche war folgende: Bei einer Anzahl von Herzleidenden verschiedener Art (Erweiterungen, Neurosen, Klappenfehlern). sowie bei einigen Herzgesunden wurden ror und unmittelbar nach dem Bade die absolute und möglichst auch die relative Herzdäinpfung durch Perkussion und Palpation. die Frequenz, Gröle, Spannung und Rhythmus des Pulses und der Blutdruck bestimmt. Das Badewasser (natürlich Süßwasser) wurde anf $35-36^{\circ} \mathrm{C}$ temperiert, den sogenannten "Indifferenzpunkt“, 111 jede thermische Wirkung auszuschliebell. Verwendet wurde der ein-, bezw. dreiphasige sinusoïdale Wechselstrom einer von der Firma Reiniger, Gebbert \& Schall bezogenen Wechselstrommaschine, die stets gleichmäligr funktionierte. Ebenfalls vol und nach dem Bade wurde die untere Lungengrenze per. kutorisch bestimmt, $11 \mathrm{~m}$ etwa durch Veränderung des Lungenvolumens hervorgerufene Tüuschıngen hinsichtlich der Herzdämpfung auszıschalten. Es fand sich in einigen Fällen eine Ansdehnung der Lungen unmittelbar nach dem Bade; diese Erscheinung beruht meines Erachtens auf dem Kältereiz der ungebenden Luft, auf die Haut beim Verlassen des lanwarmen Bades (auf die Hautoberfläche), wodurch bisweilen einige tiefere Respirationen ausgelöst werden; in wenigen Minuten war ausnahmslos die Erscheinung vorüber und die Lunge in ihre frïheren Grenzen zurückgekehrt.

Die Dauer des Bades betrug zehn Minuten.

Bei 16 Untersuchungen, angestellt an 13 Herzkranken und drei Herzgesunden, fand sich nun eine durchschnittliche Abnahme der Pulsfrequenz um vier Schläge pro Minute nach dem Bade und eine durchschnittliche Steigerung des Blutdruckes ım $8 \mathrm{~mm}$ Quecksilber. Die gröBte Abnahme der Pulszahl im einzelnen Falle war 12 Schläge; einmal (bei einem Gesun. den) nahm der Puls um 12 Schläge zu; die bedeutendste, einzeln beobachtete Drucksteigerung betrug $25 \mathrm{~mm} \mathrm{Hg}$; zweimal nahm der Blutdruck ab um 25, bezw. $5 \mathrm{~mm}$. Rechnen wir die drei Herzgesunden ab, so erhalten wir als Durchschnittszahl der Blutdrucksteigerung $8,5 \mathrm{~mm}$, und der Abnahme der Pulsfiequenz 5 pro Minute. 